\title{
More of me! Distinguishing self and reward bias using redundancy gains
}

\author{
Jie Sui ${ }^{1,2}$ • Glyn W. Humphreys ${ }^{1,3}$
}

Published online: 12 August 2015

(C) The Psychonomic Society, Inc. 2015

\begin{abstract}
Participants show a perceptual bias favoring stimuli associated with the participants themselves over stimuli associated with other people. A major account of this self-bias effect is that self-related information is intrinsically rewarding, and that high-reward stimuli have enhanced perceptual processing. Here we used redundancy gains to examine the relations between self bias and reward, and whether self and reward biases modulate common levels of stimulus integration. We demonstrated that the self-association bias increases when more than one exemplar of the stimulus is presented (i.e., when participants are exposed to redundant stimuli). The larger self-bias effects for redundant than for single stimuli arose at both perceptual and conceptual levels of representation (respectively, for identical and nonidentical stimuli associated with the same category). In contrast, high-reward stimuli did not affect perceptual redundancy gains with identical shapes, but they did affect redundancy gains with nonidentical stimuli associated with the same category. The strong redundancy effects with self-related stimuli are consistent with self associations modulating stimulus integration at both perceptual and conceptual levels, whereas reward only modulated higher-level conceptual processes (with nonidentical stimuli).
\end{abstract}

Jie Sui

jie.sui@psy.ox.ac.uk

Glyn W. Humphreys

glyn.humphreys@psy.ox.ac.uk

1 Department of Experimental Psychology, University of Oxford, South Parks Road, Oxford, UK

2 Department of Psychology and Center for Biomedical Imaging Research, School of Medicine, Tsinghua University, Beijing, China

3 Department of Speech \& Communication, Hong Kong University, Hong Kong, China
The data provide two novel theoretical advances, by showing that (i) self association modulates both early perceptual coding and higher-level conceptual coding, whereas reward only affects the higher-level process, and (ii) self bias can not be reduced simply to differential effects of reward.

Keywords Self bias · Reward bias · Redundancy gain

People show biased responses to information associated with themselves compared with other people. This bias has been found in a range of tasks, from studies of memory through to judgments of character traits and face recognition (e.g., see Cunningham, Turk, Macdonald, \& Macrae, 2008; Ma \& Han, 2010). Effects have also been demonstrated on perceptual matching, in which a self association to a shape lessens the effects of reducing stimulus contrast (luminance), as compared to other-person associations (Sui, He, \& Humphreys, 2012). These results indicate that self bias is a pervasive aspect of human information processing, modulating multiple factors, including perception as well as memory. The factors underlying the self-bias effect, though, remain poorly understood.

One major account of why self biases arise links the effects to reward. For example, Northoff and Hayes (2011) argued that self biases may come about because self-related stimuli are inherently rewarding, and that reward-based reinforcement changes both perception and memory. This argument for reward mediating self biases on cognition has empirical support. For instance, self-bias effects on perceptual matching can be mimicked by varying the reward values associated with stimuli, because stimuli associated with high reward behave similarly to stimuli associated with the self-for instance, lessening the effects of contrast (luminance) reduction on matching the shape to its associated label (Sui et al., 2012). Here we 
attempted to test whether reward was a critical driver of self bias in perceptual processing, using a redundancy gain paradigm. Redundancy gains have previously been used to test the level(s) at which the integration of information occurs (e.g., is information integrated at perceptual, conceptual, or response levels? see Miller, 1982; Mordkoff \& Yantis, 1991; Raab, 1962; Townsend \& Nozawa, 1995). We asked whether both self and reward biases enhance the gains to processing from presenting redundant stimuli. We also asked whether the two biases modulate redundancy gains at the same level of processing. To do this, we contrasted redundancy gains when the two shapes had the same physical identity and when they had different shapes but mapped onto the same conceptual identity (e.g., when square and circle stimuli presented together were both associated with the self). The same-physical-shape and the same-conceptual-identity conditions were matched, except in terms of the perceptual similarity shared by the physically identical shapes. Thus, any difference in the redundancy gains for the physically identical and conceptually identical conditions should reflect effects at a perceptual level. In contrast, if effects were due to the integration of information at conceptual and/or response levels, then redundancy effects should be the same for the physical and conceptual identity conditions, since they both mapped onto a common response (see the Method section below). By varying the relations between the redundant shapes, we could address whether the biases affect different levels of processing.

\section{Redundancy gains}

It is well established that, when we have to verify the presence of a target, performance is enhanced when two targets are present, relative to when only a single target appears (e.g., Miller, 1982; Mordkoff \& Danek, 2011). This "redundancy gain" occurs both when targets are identical and when they are nonidentical but map onto a common response (e.g., Yankouskaya, Booth, \& Humphreys, 2012). For example, in Yankouskaya et al.'s study, the task was to detect a target face, a target expression in a face, or a stimulus that combined the target identity with the facial expression. They found a redundancy gain when the target face identity and expression were combined. This result suggests that the presence of the combined features (identity and expression) in the same object enhanced the redundancy gain, by presenting observers with an emergent facial configuration (indeed, the redundancy effects decreased when the faces were inverted to reduce the saliency of the facial configuration). Similarly, other investigators have reported that redundancy gains increase when stimuli are part of a common perceptual object, and decrease when stimuli are in separate perceptual objects (Mordkoff \& Danek, 2011). Redundancy gains are also stronger when stimuli are defined by common onsets rather than offsets (Fischer
\& Miller, 2008), consistent with greater grouping between onsets than offsets (Humphreys, Riddoch, Nys, \& Heinke, 2002). On the other hand, the evidence for redundancy gains at the response level is not strong when physiological measures of motor preparation are taken (Mordkoff, Miller, \& Roch, 1996). For example, Mordkoff et al. found no effects of redundancy when response force was measured as an index of the effects on motoric processes.

Most studies have assessed redundancy gains on the basis of simple perceptual properties of the stimuli (e.g., their color or orientation; Mordkoff \& Danek, 2011; Mordkoff \& Miller, 1993), and we have sparse evidence as to whether higher-level processes modulate the effects. Evidence of higher-level factors (e.g., self-representation) modulating redundancy gains would suggest that higher-level processes can penetrate early perception (Sui et al., 2012). This was also supported by the evidence from event-related potential (ERP) studies. For example, Keyes and colleagues (2010) found that self-faces evoked stronger amplitudes of the early face N170 component than did familiar others' faces. The data suggest that selfrepresentation can modulate visual processing. We examined this here by testing whether social associations with a simple geometric shape (i.e., whether the shape is associated to you as opposed to another person) affect the magnitude of redundancy gains in shape-matching and identification tasks. We also varied whether there were redundancy gains between nonidentical shapes that mapped onto the same response ("conceptual redundancy"), or whether the gains occurred only when the shapes mapped onto the same response and were also physically identical ("perceptual redundancy"). Consider if we were to find greater gains, or a greater influence of the self or reward, on perceptual rather than conceptual redundancy. This would fit with one factor (e.g., self bias or reward) modulating perceptual processing more than the other. For example, suppose that only self bias generated larger redundancy gains between identical shapes, whereas this was not the case for reward. Here we might conclude that self bias modulates perceptual gains from redundancy but reward does not, and hence that the self and reward are distinct concepts.

\section{Self biases in perception}

Self biases have previously been observed in tasks addressing different levels of processing. For example, memory is enhanced when participants make judgments about selfreference (Rogers, Kuiper, \& Kirker, 1977; Symons \& Johnson, 1997) and self-ownership, as compared with when they make similar judgments in relation to other people (e.g., Cunningham et al., 2008). Participants are also faster to make familiarity decisions to images of their own faces than to the faces of other people in face perception tasks (Keenan et al., 1999; Keyes \& Brady, 2010; Sui \& Humphreys, 2013), and self-related information (hearing your own name, or seeing 
your own face) can capture attention (Brédart, Delchambre, \& Laureys, 2006; Moray, 1959; Tong \& Nakayama, 1999). However, with stimuli such as our own faces or own names, it is difficult to rule out effects of stimulus familiarity on performance, since we are likely to be more familiar with our own face and name than with the faces and names of other people. Hence, it is difficult to deduce whether self-related information is special or just more familiar. It is also difficult to explore whether the self biases reflect changes in perceptual processing or responding, and whether self biases stem from changes in more basic "drivers" of performance-for example, because self-related information has high intrinsic reward value (Northoff \& Hayes, 2011).

In order to address these issues, we recently developed a new procedure for examining self (and, indeed, other social) biases on perception (Sui et al., 2012, 2013). In this procedure, participants associate a shape with a label that can be selfrelated (e.g., square-you), related to a familiar other (e.g., circle-friend), or related to a stranger (e.g., triangle-other). They are then asked to decide whether shape-label pairs are as originally presented (square-you, circle-friend) or are re-paired (square-friend, circle-you). Participants are much faster and have enhanced perceptual sensitivity when verifying selfrelated matched pairs (square-you) than with associations involving other people (circle-friend, triangle-other). Note that, since neutral shapes are used, effects of differential familiarity with particular stimuli can be factored out. In addition, we showed similar effects when participants made associations to low versus high reward values, suggesting that self biases may be linked to high intrinsic reward (Sui et al., 2012).

\section{The present study}

Here we exploited the self-association procedure introduced by Sui et al. (2012), to examine whether self bias modulated perceptual processes sensitive to redundancy gains. In Experiments 1 and 2, participants learned two shape associations relative to the self or to a best friend. We then presented either single shapes or shape pairs, and participants had to identify whether the shapes were associated with a particular individual; on redundant trials, the shapes could be identical or nonidentical (but were associated with the same person). We tested whether redundancy gains were enhanced for selfassociated stimuli relative to stimuli associated with other people, and whether effects were found with both identical and nonidentical shapes (at perceptual and higher-level conceptual levels, respectively). Note that, with nonidentical shapes, redundancy gains may stem from faster access to associations formed at a relatively abstract, conceptual level, and also from effects on decision making. Effects with physically identical shapes, however, might reflect perceptual grouping in addition to any conceptual and response effects (see the General Discussion for more consideration of this point).
Experiments 3 and 4 generalized the procedures to the effects of reward (Northoff \& Hayes, 2011). Would the effects of reward in Experiments 3 and 4 match those of self association observed in Experiments 1 and 2-using a similar paradigm? Would the effects of reward be manifest for both perceptual and conceptual redundancy gains? Note that evidence for a dissociation between the effects of self and reward (e.g., due to their influencing different levels of redundancy gain) would suggest that the two effects are driven by distinct underlying mechanisms.

\section{Self and redundancy gains}

\section{Experiment 1: name-shape matching}

Participants were verbally told the different shape-label associations (e.g., "you are represented by a circle and a square") and then performed the matching task. In Experiment 1, the person's name was first presented and followed by one or two shapes that could match or mismatch the name. We asked whether presenting redundant stimuli would facilitate performance relative to when only a single shape appeared, and whether this varied according to whether the shapes were (i) physically identical and (ii) associated with the self.

\section{Method}

Participants There were 27 Chinese college students (17 female, ten male; 19 to 36 years of age, $M \pm S D=23.07 \pm 4.46$ ). All of the participants were right-handed and had normal or corrected-to-normal vision. Informed consent was obtained according to procedures approved by a local ethics committee.

Stimuli and tasks Six geometric shapes (circle, hexagon, rectangle, ellipse, square, and triangle) were randomly assigned to three conditions. Two shapes were associated with the self, two were associated with the gender-matched best friend of the participant (whom the participant named), and two were used as neutral baseline stimuli (represented as "none"). For mismatched trials, the self or friend name was independently paired with the baseline shape(s) (on two-item trials, the neutral shapes were identical or different; on singleitem trials, just one neutral shape appeared). There were no mixed self-friend pairs. Participants were instructed to respond whether the name initially presented was matched by at least one person-associated shape. A name cue, presented at $2.2^{\circ}-3.2^{\circ} \times 2.0^{\circ}$ in size, was displayed at the center of the screen, followed by one or two shape stimuli, each subtending $3.8^{\circ} \times 3.8^{\circ}$, in the left or/and right visual fields. The distance between the center of the shape and the central fixation cross (itself $0.8^{\circ} \times 0.8^{\circ}$ ) was $3.0^{\circ}$. The background was gray. The experiment was run on a PC using E-Prime software (Version 
$1.1)$ and was displayed on a 19 -in. monitor $(1,024 \times 768$ at $60 \mathrm{~Hz})$.

Procedure The experiment had two stages. First, participants were asked to code two geometric shapes as a named best friend, two as the self, and two as neutral stimuli. This pretest lasted just 60 s (see Sui et al., 2012). For example, a participant might be told, "The triangle and hexagon represent your best friend Mary; the circle and rectangle represent you; and the ellipse and square will represent neutral stimuli ('none')." The shapes themselves were not presented at this stage. Subsequently, a sequential name-shape matching task was carried out. Participants judged whether, after a name was presented, the following display contained a matching shape. On each trial, a central cross appeared for $500 \mathrm{~ms}$, followed by a name (self or friend) for $100 \mathrm{~ms}$. Subsequently, a variable blank time window was presented for a duration ranging from 100 to $300 \mathrm{~ms}$. After this, one or two shapes were presented in the left and/or right visual field for $100 \mathrm{~ms}$. For match trials a name-shape pairing could conform to those instructed, or for mismatched trials the pairing could be a recombination of a name with a neutral or a different shape (or shapes, on twoitem trials). A variable-duration blank interval then followed (ranging from 900 to $1,100 \mathrm{~ms}$ ). Participants had to press one of two response buttons as quickly and accurately as possible within this time frame (to encourage immediate responding). Feedback ("correct" or "incorrect") appeared on the screen for $500 \mathrm{~ms}$ at the end of each trial.

Each participant carried out three blocks of 240 trials following eight practice trials, in which self, friend, and re-paired self/friend-neutral stimuli occurred equally often, in a random order. The accuracy of performance after each block was reported. Thus, three within-subjects variables were testedmatching trial (Match: matching vs. mismatching name-shape pairs), priming names (Association: friend vs. self), and the number of test shapes (Shape: single, two same, or two different shapes). There were 60 trials in each of the 12 conditions across the three factors. The shapes assigned to the self, friend, and neutral conditions were counterbalanced across participants.

We report effect sizes using partial eta-squared $\left(\eta_{\mathrm{p}}{ }^{2}\right)$ for analyses of variance (ANOVAs) and Cohen's $d_{\mathrm{z}}$ for all $t$ tests (Lakens, 2013). Holm-Bonferroni corrections for $\alpha=.05$ were applied to all multiple comparisons (Holm, 1979).

\section{Results and discussion}

The accuracy data on matched and mismatched trials were used to derive a measure of perceptual sensitivity $(d$; see Fig. 1a). These data followed the pattern of the reaction time (RT) data (Fig. 1b) and were not analyzed further. RTs were analyzed using a three-way repeated measures ANOVA with the factors Association (friend vs. self), Shape (single, two same or different shapes), and Match (matched vs. mismatched). The results revealed a significant main effect of shape, $F(2,52)=7.96, p=.001, \eta_{\mathrm{p}}^{2}=.23$; Paired simple $t$ tests showed that participants had faster responses on trials with two physically identical (same) shapes $[t(26)=-2.54, p$ $\left.=.018, d_{\mathrm{z}}=0.49\right]$ or with two conceptually identical (different) shapes $\left[t(26)=-2.67, p=.013, d_{\mathrm{z}}=0.51\right]$ than in the one-shape baseline, reflecting a general redundancy gain, but no overall difference was apparent between same- and different-shape trials $[t(26)=0.85, p=.41]$. There was also a significant main effect of match, $F(1,26)=9.64, p=.005$, $\eta_{\mathrm{p}}{ }^{2}=.27$; responses were faster to match than to mismatch trials. The effect of association was not significant, $F(1,26)=$ $3.30, p=.08$. These effects were qualified by a significant three-way interaction between shape, match, and association, $F(2,52)=5.92, p=.005, \eta_{\mathrm{p}}{ }^{2}=.19$ (Fig. 1b). We conducted ANOVAs with Association and Shape as factors for the match and mismatch conditions, respectively. For match trials, we conducted two ANOVAs - one using raw scores in order to follow the above analysis, and the other using relative scores between the two-shape and single-shape conditions (indexing the size of the redundancy gains) in order to directly examine whether redundancy gains were modulated by self biases.

Analysis of the raw sores revealed a significant interaction between association and shape, $F(2,52)=4.17, p=.02, \eta_{\mathrm{p}}{ }^{2}=$ .14. Larger self biases (i.e., faster responses relative to the friend condition) were associated with trials with two physically identical (same) shapes, $t(26)=-3.00, p=.006, d_{\mathrm{z}}=$ 0.57 . There were also faster responses on different-shape trials in the self than in the friend condition, $t(26)=2.47, p=.02, d_{\mathrm{z}}$ $=0.49$. We found no difference between the self and friend associations on single-shape trials, $t(26)=1.54, p=.14$ (Fig. 1c). The analysis of the relative scores showed a significant interaction between association (friend vs. self) and the redundancy gain (two different vs. two same shapes), $F(1,26)$ $=6.44, p=.02, \eta_{\mathrm{p}}{ }^{2}=.20$. Paired-samples $t$ tests revealed an enhanced redundancy gain for the self over the friend association when there were two identical shapes, $t(26)=2.40, p=$ $.02, d_{\mathrm{z}}=0.47$, whereas no difference in redundancy gains was apparent for self and friend stimuli with two different shapes, $t(26)=0.96, p=.35$ (Fig. 1d).

In the mismatch condition, we observed a significant main effect of shape, $F(2,52)=4.45, p=.02, \eta_{\mathrm{p}}{ }^{2}=.15$; faster responses were registered to two same shapes than to a single shape, $t(26)=-3.53, p=.002, d_{\mathrm{z}}=0.69$, and to two different shapes than to a single shape, $t(26)=-2.43, p=.02, d_{\mathrm{z}}=0.46$, but no difference was apparent between two same and two different shapes, $t(26)=0.25, p=.80$. Neither the main effect of association nor the interaction between association and shape was significant, $F(1,26)=0.04, p=.84$, and $F(2,52)$ $=2.72, p=.08$.

These results indicate that newly established personal associations modulate redundancy gains in label-shape 


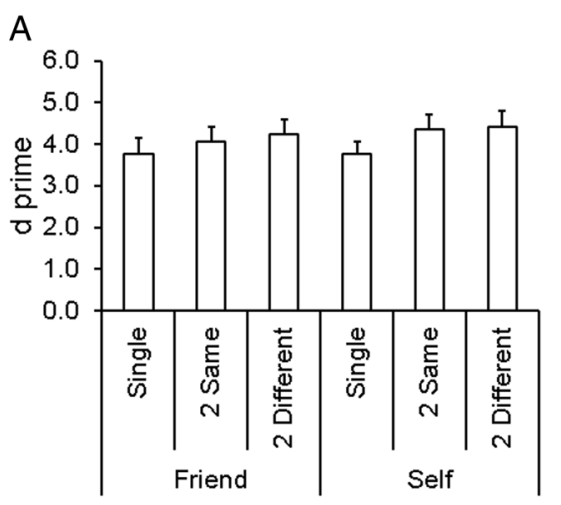

B

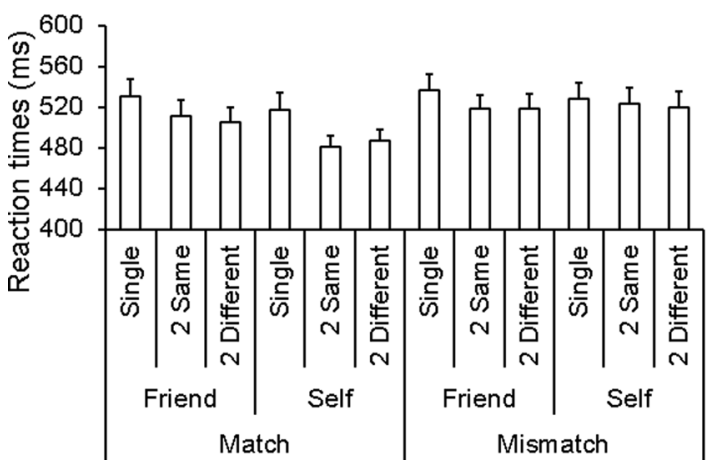

C

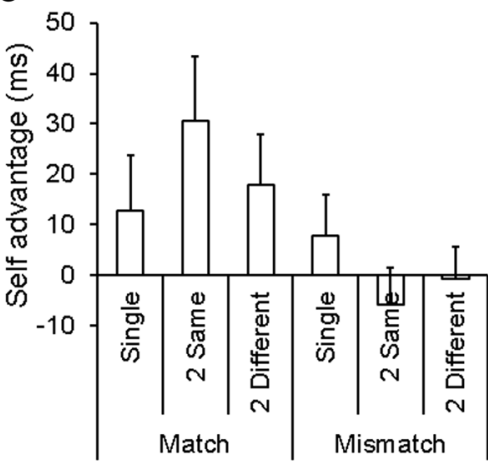

D

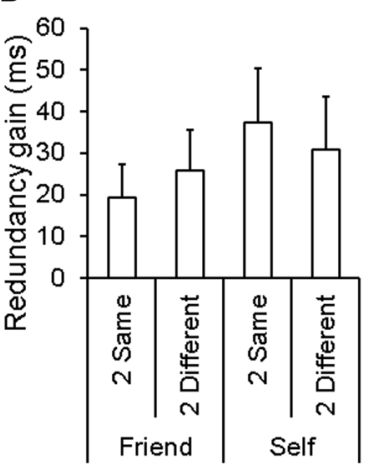

Fig. 1 Performance in Experiment 1. a Perceptual sensitivity $\left(d^{\prime}\right)$ as a function of association (friend vs. self) and shape (single, two different, or two same shapes). b Mean reaction times (RTs) as a function of association (friend vs. self), shape (single, two different, or two same shapes), and match (match vs. mismatch). c Magnitude of the self advantage (vs. responses to friend-associated stimuli) in RTs, as a

matching. Matching responses were facilitated on trials with two relative to one shape, with the effect being most pronounced when the shapes were physically identical, as compared to when they were nonidentical but mapped onto the same concept (self or friend). This effect was enhanced for self-related stimuli in the conditions with two physically identical shapes. This enhancement of the redundancy effect is consistent with the self-related association boosting perceptual integration between the stimuli (see the General Discussion for more consideration of this point). To establish the robustness of this result, a replication was sought in Experiment 2.

In Experiment 1, participants saw the name before the shapes. This presentation format may encourage top-down expectancies of the stimuli, so self biases might have been particularly prominent under these top-down, predictive conditions. Sui, Sun, Peng, and Humphreys (2014) have recently examined self biases on perceptual matching when the probabilities of self-, friend-, and other-related matching stimuli were varied. The self-bias effect was robust across different probability relations, suggesting a relatively automatic component to the effect. However self biases also increased when self-match stimuli appeared with a high probability, indicating that the effect could be enhanced when participants formed a function of the shape (single, two different, or two same shapes) and match condition (match vs. mismatch). d Redundancy gains (two stimuli vs. a single stimulus) as a function of association (friend vs. self) and shape (two different vs. two same shapes). Error bars represent standard errors

top-down expectancy for self-related stimuli. Experiments 2 and 3 were designed to rule out top-down effects on performance by eliminating the initial stimulus cue on the trial.

\section{Experiment 2: shape judgments to stimuli}

In Experiment 2, participants underwent the same initial association process as in Experiment 1, but only for four shapes: Two were associated with the self, and the other two with a best friend. After the association instruction, participants received the shape display (there were now no name primes), and they had to identify whether the display contained a shape associated with the self or with a friend. Participants were presented with single-item displays (friend vs. self) and twoitem displays (same-shape friend, different-shape friend, same-shape self, or different-shape self). Since the stimulus cue was not presented before the shapes, the opportunity for top-down expectancies being formed was reduced.

\section{Method}

A total of 20 Chinese college students participated (13 female, seven male; 20 to 24 years of age, $M \pm S D=21.60 \pm 1.05$ ). 
Experiment 2 was identical to Experiment 1, except that no name cue was presented before the shape stimuli, and the task was to judge whether one or two shapes presented in the left and/or right visual fields represented the self or the friend. We manipulated two within-subjects factors-Association (friend vs. self) and Shape (single, two different, or two same shapes).

\section{Results and discussion}

The accuracy analysis again showed no evidence of a speedaccuracy trade-off (Fig. 2a and b). The ANOVAs for RTs showed a significant main effect of association, $F(1,19)=$ $5.71, p<.03, \eta_{\mathrm{p}}{ }^{2}=.23$; participants had faster responses to the self association than to the friend association, indicating biased responses to the self. We also observed a significant main effect of shape, $F(2,38)=25.86, p<.001, \eta_{\mathrm{p}}{ }^{2}=.58$, indicating the presence of redundancy gains; there were faster responses to two same $\left[t(19)=-6.68, p<.001, d_{\mathrm{z}}=1.45\right]$ and to two different $\left[t(19)=-5.47, p<.001, d_{\mathrm{z}}=1.15\right]$ shapes than to single-shape trials. Same- and different-shape trials did not differ $[t(19)=-0.30, p=.77]$. The interaction was also significant, $F(2,38)=3.39, p<.05, \eta_{\mathrm{p}}{ }^{2}=.15$ (Fig. 2b). Planned pairwise $t$ tests showed faster responses to the self than to the friend trials with two different shapes, $t(19)=2.78, p=.012$, $d_{\mathrm{z}}=0.60$, as well as an advantage for self over friend trials with two identical shapes, $t(19)=2.19, p=.04, d_{z}=0.52$. There was no difference between the responses to self and friend stimuli on single-item trials, $t(24)=0.75, p=.46$ (Fig. 2c). In line with the results of Experiment 1, in order to test whether self biases can affect redundancy gains, we also conducted another ANOVA using relative scores (the difference between the single-shape and two-shape conditions) with two factors: Association (friend vs. self) and Shape (two different vs. two same shapes). We found a significant main effect of association, $F(1,19)=9.60, p<.01, \eta_{\mathrm{p}}{ }^{2}=.33$, with a larger redundancy gain for self than for friend associations. No significant main effect of shape was apparent, however, and no interaction, $p \mathrm{~s}<.51$ (Fig. 2d). The data indicate that a self bias modulates redundancy gains at both perceptual and conceptual levels.
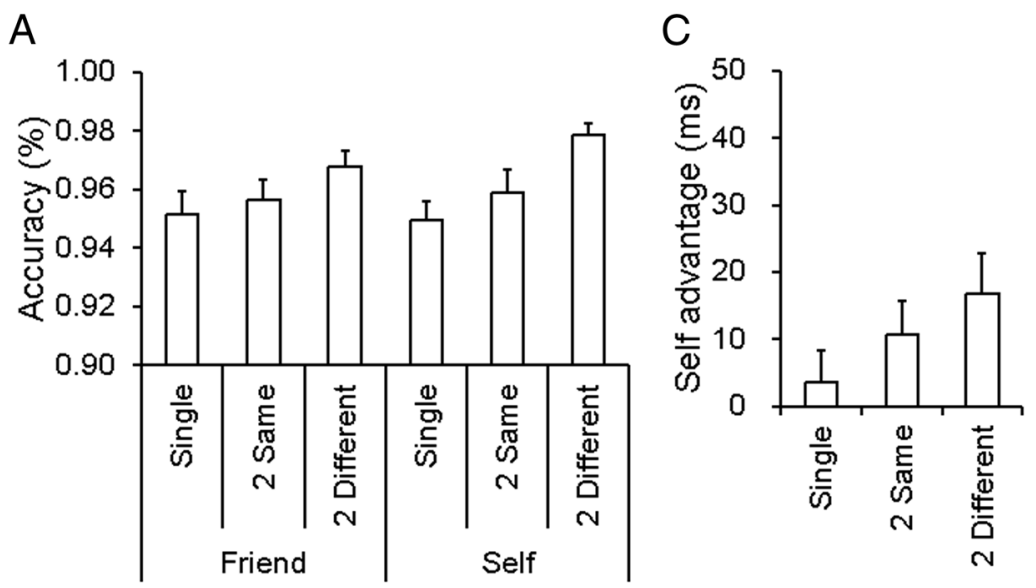

B

D
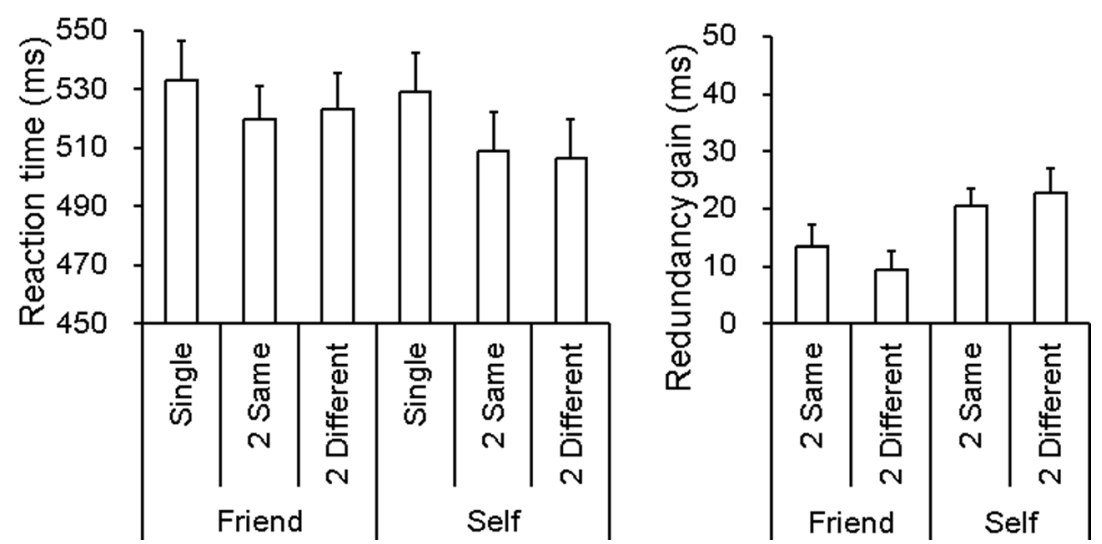

Fig. 2 Performance in Experiment 2. a Mean accuracy (proportions) as a function of association (friend vs. self) and shape (single, two different, or two same shapes). b Mean reaction times (RTs) as a function of association (friend vs. self) and shape (single, two different, or two same shapes). c Magnitude of the self advantage (vs. responses to

friend-associated stimuli) in RTs, as a function of the shape condition (single, two different, or two same shapes). d Redundancy gains (two stimuli vs. a single stimulus) as a function of association (friend vs. self) and shape (two different vs. two same shapes). Error bars represent standard errors 


\section{Cross-experimental analyses between experiments 1 and 2}

In order to examine the effect of top-down cues on redundancy gains, we compared the redundancy gains in Experiments 1 and 2. We conducted ANOVAs with one between-subjects variable-Experiment (1 vs. 2) - and two within-subjects variables-Association (friend vs. self) and Redundancy Gain (two different vs. two same shapes). The results revealed a significant main effect of association, $F(1,45)=8.16, p=.006, \eta_{\mathrm{p}}{ }^{2}=.15$ : There was a larger redundancy gain in the self- than in the friendassociation condition. The effects of redundancy gain (two different vs. two same shapes) and experiment were not significant, $F(1,45)=0.001, p=.98$, and $F(1,45)=0.51, p=.48$, respectively. No significant interactions emerged involving experiment, $F_{\mathrm{s}}<3.71, p \mathrm{~s}>.06$. The failure to find any interaction with experiment indicated that there were comparable self-bias effects on the redundancy gains in Experiments 1 and 2. These data suggest that top-down influences may not be critical to generate self biases for redundancy gains.

\section{Reward and redundancy gains}

Having demonstrated a pattern of redundancy gains for selfassociated stimuli, in Experiments 3 and 4 we set out to assess redundancy gains for reward-related items. In Experiment 3 we used the shape-matching procedure employed in Experiment 2, and in Experiment 4 we used the name precue procedure from Experiment 1, in order to enhance any possible top-down influence for reward-related shapes.

\section{Experiment 3: shape judgments to stimuli}

\section{Method}

There were 23 Chinese college students ( 18 female, five male; 20 to 26 years of age, $M \pm S D=23.09 \pm 2.30$ ). Experiment 3 was identical to Experiment 2, except that participants were asked to form low- versus high-reward value associations to each of two shapes ( $¥ 1$ vs. ¥9)—a procedure previously shown to modulate perceptual-matching performance (Sui et al., 2012). Participants were instructed to judge whether one or two shapes presented in the left and/or the right visual field represented the high or low reward value. Participants were told that they would be rewarded extra differential bonus funds according to their correct responses to high- and lowreward shapes, in addition to being given a participation fee. The maximal account was $¥ 9$ plus $¥ 1$, and each correct response was awarded $¥ 9 / ¥ 1 \times 1 \%$. We manipulated two within-subjects factors-Association (low vs. high reward) and Shape (single, two same, or two different shapes).

\section{Results and discussion}

The accuracy performance showed the same trends as the RT data (Fig. 3a and b) and was not analyzed further. The ANOVAs for RTs showed a significant main effect of shape, $F(2,44)=8.80, p=.001, \eta_{\mathrm{p}}{ }^{2}=.29$, with faster responses in the two-same $\left[t(22)=-4.71, p<.001, d_{\mathrm{z}}=0.92\right]$ and the twodifferent $\left[t(22)=-2.83, p=.01, d_{\mathrm{z}}=0.56\right]$ shape conditions than in the single-shape condition, but similar overall performance for same- and different-shape trials $[t(22)=0.31, p=$ .76]. The effect of association was not significant $[F(1,22)=$ $1.47, p=.24]$, but the interaction of shape and association was, $F(2,44)=8.03, p=.001, \eta_{\mathrm{p}}{ }^{2}=.27$ (Fig. 3b). Pairwise $t$ tests showed an advantage for high- over low-reward stimuli when two different shapes appeared, $t(22)=-3.30, p=.003, d_{\mathrm{z}}=$ 0.66 , but not for trials with two same or with single shapes, $t \mathrm{~s}(22)=-0.25$ and $0.03, p \mathrm{~s}=.81$ and .97 (Fig. $3 \mathrm{c}$ ).

We then tested the level (perceptual vs. conceptual) at which high-reward biases modulated the redundancy gains, by conducting ANOVAs using the relative scores between the single- and two-shape conditions with two factors-Association (low vs. high reward value) and Shape (two different vs. two same shapes). A significant main effect of association, $F(1,22)=5.95, p=.02, \eta_{\mathrm{p}}^{2}$ $=.21$, indicated a greater redundancy gain in the highthan in the low-reward condition. There was also a marginally significant interaction, $F(1,22)=4.85, p=.038$, $\eta_{\mathrm{p}}{ }^{2}=.18$. The interaction reflected a larger redundancy gain for the high-reward than for the low-reward condition for the different-shape trials, $t(22)=-3.55, p=$ $.002, d_{\mathrm{z}}=0.75$, whereas there was no effect of reward value with two identical stimuli, $t(22)=-0.11, p=.62$ (Fig. 3d).

The data in Experiment 3 replicated the results from Sui et al. (2012) in showing facilitated processing of shapes associated with high reward relative to those associated with low reward. The new result is that this highreward advantage only emerged on trials with different shapes mapping onto the same value. In the case of physically identical shapes, the high-reward advantage seemed to have been subsumed within the advantage from perceptually grouping two identical shapes. In contrast, a self advantage had remained for identical shapes in both Experiments 1 and 2. The presence of a self bias and the absence of a reward bias on same-shape trials was not due to the smaller magnitude of the reward bias than of the self bias, since the high-reward advantage with different shapes was comparable to the magnitude of the self advantage in Experiment 2 (using the same paradigm; $16.70 \pm 6.01 \mathrm{~ms}$ for the self advantage vs. $17.03 \pm$ $5.36 \mathrm{~ms}$ for the high-reward advantage), $t(41)=-0.07, p$ $=.95$. This differential effect highlights a qualitative contrast between the effects of reward and of self. 


\section{A}

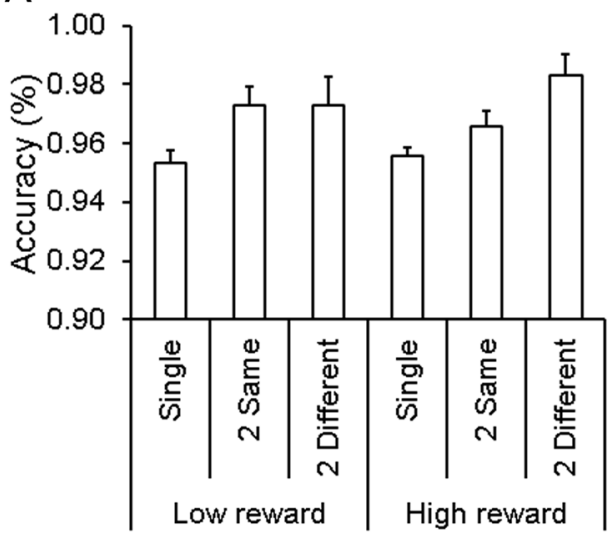

$\mathrm{B}$

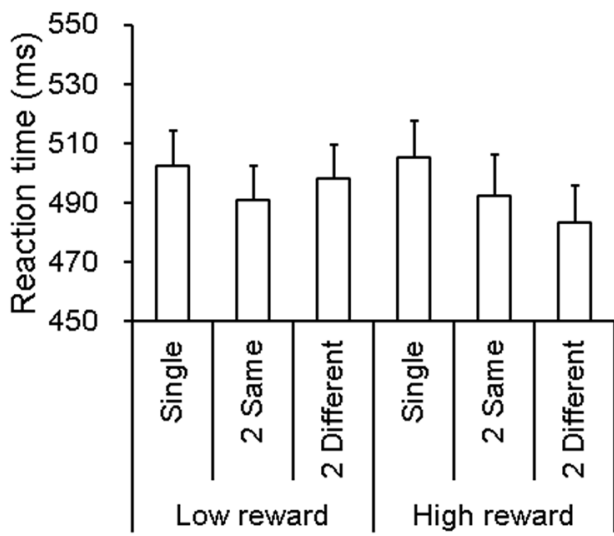

C

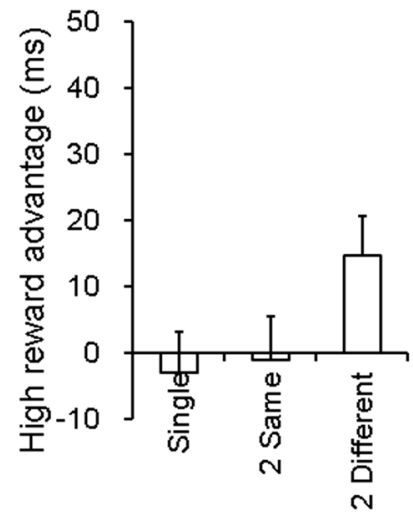

D

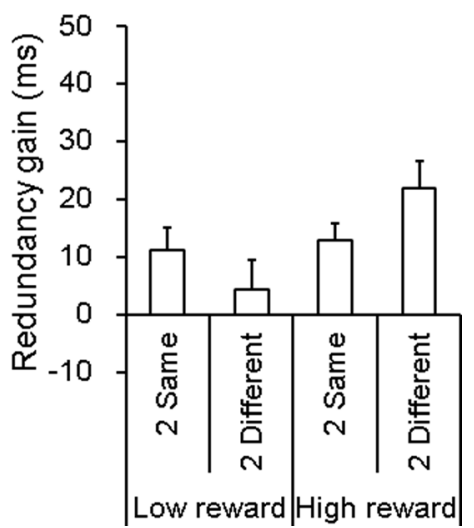

Fig. 3 Performance in Experiment 3. a Mean accuracy (proportions) as a function of association (low vs. high reward) and shape (single, two different, or two same shapes). b Mean reaction times (RTs) as a function of association (low vs. high) and shape (single, two different, or two same shapes). c Magnitude of the high-reward advantage (vs.

\section{Experiment 4: name-shape matching}

In Experiment 3, the reward value that was linked to the shapes influenced the redundancy gains when the two shapes on a trial were different, but not when they were the same. In Experiment 4, we only focused on the condition with two identical shapes, in order to optimize the association effects in this condition. In Experiment 4, we used the cue word procedure from Experiment 1 in order to try to enhance reward-based redundancy gains. For the study, we presented a high or a low reward value followed by the associated shape stimuli, and participants had to judge whether the shapes presented after the cue matched the cue word. In line with Experiment 1, this procedure should encourage top-down expectancies to be formed for the shape associated with the cue, optimizing matching performance and the reward-based modulation of redundancy gains. In addition, two other changes were made, as compared with Experiment 1. One was that we had participants form just one shape association to one reward. This meant that we eliminated the different-shape redundancy responses to low-reward-associated stimuli) in RTs, as a function of the shape condition (single, two different, or two same shapes). d Redundancy gains (two stimuli vs. a single stimulus) as a function of the association (low vs. high reward) and shape (two different vs. two same shapes). Error bars represent standard errors

condition, but it also meant that learning of the reward-shape association was maximal. The second change was that we increased the differential between the high and low reward values, from the high reward being 9 times greater (Exp. 3) to 16 times greater (Exp. 4), to enhance the effects of the reward association on shape matching. In line with Experiment 3, participants were rewarded an extra differential bonus based on their correct responses to high- and lowreward shapes, in addition to being given a participation fee.

\section{Method}

For this experiment, we recruited 19 British college students (15 females, four male; 20 to 23 years of age, $M \pm S D=20.74$ \pm 0.93 ). Experiment 4 was identical to Experiment 1, except that participants were asked to form low- versus high-reward value associations to one of two shapes ( $£ 0.5$ vs. $£ 8$; Sui et al., 2012). Two shapes were associated with the high and low reward values, respectively. For matched trials, a cue indicating high or low reward ( $£ 0.5$ or $£ 8$ ) was presented 
followed by the high- or low-reward associated shape(s) (two identical items or single items). On mismatch trials, the cue was followed by the nonassociated shape (high-reward cuelow-reward shape, or vice versa). Participants were instructed to respond according to whether the reward value matched the shape(s). Participants were rewarded extra differential bonus funds based on their correct responses to high- and lowreward shapes, in addition to being given a participation fee, with each correct response being awarded either $£ 0.5$ or $£ 8 \times$ $1 \%$ (for correct responses to the low- and high-reward stimuli, respectively). We manipulated three within-subjects factors-Matching Trial (matching vs. mismatching value-shape pairs), Priming Value (low vs. high), and Number of Test Shapes (single vs. two same shapes).

\section{Results and discussion}

The accuracy data on matching and mismatching trials were used to derive a measure of perceptual sensitivity ( $d$ '; see Fig. 4a) and were not analyzed further, since they followed the pattern of the RT data (Fig. 4b). RTs were analyzed with three within-subjects factors: Association (low vs. high), Shape (single vs. two same shapes), and Match (matched vs. mismatched trials). The ANOVAs revealed significant main effects of shape and match, $F(1,18)=6.19, p<.03, \eta_{\mathrm{p}}{ }^{2}=.26$,

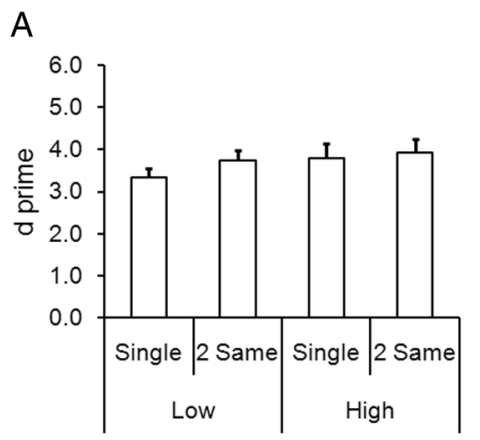

B

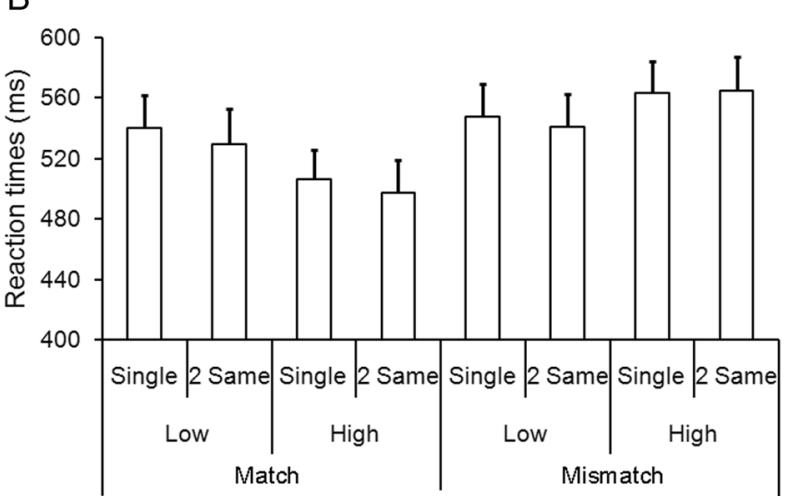

Fig. 4 Performance in Experiment 4. a Perceptual sensitivity $\left(d^{\prime}\right)$ as a function of association (low vs. high) and shape (single vs. two same shapes). b Mean reaction times (RTs) as a function of association (low vs. high), shape (single vs. two same shapes), and match (match vs. mismatch). c Magnitude of the high-reward advantage (vs. responses to

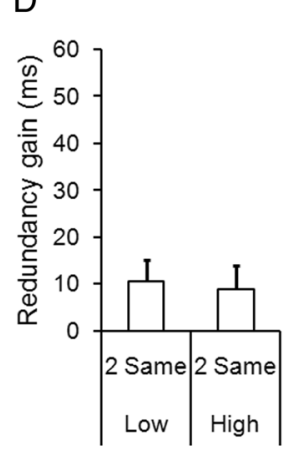

and $F(1,18)=20.72, p<.001, \eta_{\mathrm{p}}{ }^{2}=.54$ : There were faster responses to trials with two rather than one shape, indicating an overall redundancy gain, and participants made more rapid responses to match than to mismatch trials. We did not observe a significant main effect of association, $F=2.15, p=.16$. No high-order interactions were significant, $F_{\mathrm{s}}<3.57, p \mathrm{~s}>$ 0.08 , except for a significant two-way interaction of association and match, $F(1,18)=18.46, p<.001, \eta_{\mathrm{p}}{ }^{2}=.51$ (Fig. 4b). Pairwise $t$ tests reflected faster responses to high than to low reward on match trials, $t(18)=-3.40, p=.003, d_{\mathrm{z}}=0.76$, but slower responses to high than to low reward on mismatch trials, $t(18)=4.12, p=.001, d_{\mathrm{z}}=1.09$.

In order to make a comparison with the self-bias effects, we measured high-reward biases (RTs to lowreward stimuli minus those to high-reward stimuli) in an ANOVA with two factors-Match (match vs. mismatch) and Shape (single vs. two same shapes). This showed a significant main effect of match, $F(1,18)=18.46, p<$ $.001, \eta_{\mathrm{p}}{ }^{2}=.51$, due to larger high-reward biases on match than on mismatch trials. The effect of shape and the interaction between match and shape were not significant, $F_{\mathrm{S}}<1.1, p \mathrm{~s}>.31$ (Fig. 4c). Thus, the effects of reward did not increase on redundant versus single-item trials. We also computed the magnitude of the redundancy gain (RTs on one-item trials minus RTs on two-item trials;

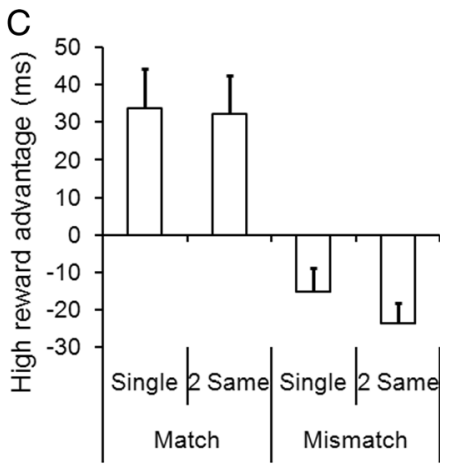

low-reward-associated stimuli) in RTs as a function of shape (single vs. two same shapes) and match condition (match vs. mismatch). d Redundancy gains (two stimuli vs. a single stimulus) as a function of association (low vs. high) in the two-same-shape condition. Error bars represent standard errors 
Fig. 4d). A paired-sample $t$ test revealed no significant difference in redundancy gains between the low-reward and high-reward associations, $t(18)=0.26, p=.80$.

These results indicate that the reward associations did not modulate redundancy gains in a shape-label matching task. This occurred even though we maximized the chances for topdown modulation by cueing the associated shape before each trial, by using only single shape associations with each reward value, and by increasing the contrast between low- and highreward values (as compared with Exp. 3). These factors did enhance the effects of the reward bias itself, and the overall reward advantage on match trials was numerically larger than the self advantage in Experiments 1 and 2 and the reward advantage in Experiment 3 (here, $>30$ ms; Fig. 4c). The lack of enhancement of the redundancy gain with identical shapes also matched the data from Experiment 3 and indicated that reward does not modulate the perceptual integration of shapes, whereas that is the case for self associations (Exps. 1 and 2).

\section{Cross-experimental analyses between experiments 3 and 4}

A comparison between Experiments 3 and 4 was conducted in order to test the effects of top-down cues on perceptual redundancy gains (two same shapes vs. a single shape) for rewardrelated stimuli. An ANOVA was conducted with one betweensubjects factor-Experiment (3 vs. 4) - and one withinsubjects factor-Association (low vs. high reward) - with the redundancy gains as the dependent variable. This revealed significant main effects of neither association nor experiment, $F_{\mathrm{s}}(1,40)<0.07, p \mathrm{~s}>.79$; the interaction between experiment and association was also not reliable, $F(1,40)=0.02, p=.90$. The failure to find any significant effects indicated that reward did not modulate the perceptual redundancy gains in Experiments 3 and 4.

\section{General discussion}

We have reported four experiments that showed that (i) both self and high-reward associations facilitate stimulus matching and identification, and (ii) self biases modulate redundancy gains with both identical and nonidentical stimuli, whereas (iii) high reward differentially affects performance with nonidentical items, but not with identical stimuli. These data highlight an important contrast between the effects of self and reward associations on information processing, and they also provide new information on how self-based information and reward modulate decision making.

Researchers have argued that self biases in information processing may stem from differential mechanisms of reward (Behrens, Hunt, Woolrich, \& Rushworth, 2008; Northoff \& Hayes 2011). For example, self-related information may have high intrinsic reward value, and this may in turn modulate perception and attention (Sui et al., 2012). Consistent with this, we found evidence that both self and reward associations can produce similar effects on performance (Anderson \& Yantis, 2013; Chelazzi et al., 2014; Chelazzi, Perlato, Santandrea, \& Della Libera, 2013). For example, Anderson and Yantis trained participants to learn associations between stimuli and different reward values, and then presented the stimuli as distractors in a visual search task. Distractors associated with high reward attracted attention away from the targets (see also Chelazzi et al., 2014). Sui et al. (2012) compared the effects of self association with the effects of tagging shapes to have different reward values. Perceptual matching was faster and more accurate for both self-associated shapes and shapes that were linked to high reward, and both types of newly formed association led to decreased effects of stimulus contrast reduction (luminance) on perceptual discrimination. These results suggest that the self bias in behavior may reflect increased reward values linked to the self. In addition, both self-related stimuli and reward modulate regions of ventromedial prefrontal cortex (Behrens et al., 2008; Hickey, Chelazzi, \& Theeuwes, 2010; Izuma, Saito, \& Sadato, 2008; Northoff \& Bermpohl, 2004; Rushworth, Noonan, Boorman, Walton, \& Behrens, 2011; Sui, Rotshtein, \& Humphreys, 2013), consistent with reward being the "driver" of self bias.

The present results contradict the strong version of this assertion - namely, that differential reward is the sole driver of self bias. Here we found that self associations affected redundancy gains with both physically identical shapes and conceptually associated shapes. In contrast, redundancy gains with physically identical shapes were unaffected by the reward values associated with the shapes, though effects with conceptually associated shapes were larger if the shapes were linked to high reward. The redundancy gains with physically identical shapes, which are greater than those with conceptually associated shapes, may reflect those perceptual processes recruited when the shapes are identical, such as perceptual integration and grouping of information between the shapes (see below for further discussion), in addition to any effects in the later stages of information processing, such as access to conceptual representations of the stimuli and response selection and execution (Mordkoff \& Danek, 2011). Our results also indicate that the integration process at the perceptual level was modulated by self bias but not by reward. Note that this did not occur because the effects of reward were intrinsically weaker; they were as strong as the self-bias effect with conceptually associated stimuli. The data point to a qualitative difference, at least between aspects of self bias and reward. They also point to a limitation of the effects of reward on information processing.

The literature on the effects of reward on information processing has indicated that perception can be affected by reward; for example, visual search is facilitated when targets have high reward (e.g., Kristjánsson, Sigurjónsdóttir, \& 
Driver, 2010). This is also supported by neuroimaging studies (e.g., Serences, 2008; Shuler \& Bear, 2006). For example, a reward manipulation can down-regulate brain activity in early visual cortex, including V1 (e.g., Serences, 2008). The present results, though, indicate that specific aspects of perceptual processing, relating to the integration of perceptual information, may be relatively immune to the effects of reward. There are limitations on which form of perceptual processing is penetrated by reward. Nevertheless, reward did affect redundancy gains at a conceptual level, when two different shapes were presented. One account of this is that reward exerts top-down modulation in the integration of information, which modulates performance at a conceptual rather than a perceptual level. The result is consistent with prior work showing that reward bias affected performance through a top-down motivation mechanism (Chelazzi et al., 2013; Hickey et al., 2010). However, the contrast with self-bias effects, which modulated redundancy gains even with physically identical shapes, indicates that at least some aspects of self bias are distinct from the effects of reward. We concluded that the effects of the self cannot simply be reduced to the effects of differential reward (de Greck et al., 2010).

Different accounts of the effects of the self on redundancy gains with identical shapes should be considered. One account, which fits with prior data on the greater redundancy gains for features on the same rather than on different objects (Mordkoff \& Danek, 2011) is that self association can help group together visual elements on the basis of the similarity of the physical properties of the stimuli (e.g., physically identical shapes, in the present study). This is consistent with the evidence from previous studies showing that high-level factors can permeate early perception (Chelazzi et al., 2013; Sui et al., 2012). We might think of self-representation being a strong glue that leads to easy assimilation and the rapid establishment of links between different elements. Here, by virtue of them both being associated with the self, a rapid linkage facilitated grouping based on physical identity, in addition to any effects based on conceptual overlap between the stimuli (i.e., when two different shapes related to the common concept of the self or a friend). It is interesting that, in the memory domain, there is evidence that better associations are formed between items that are related to the self than between those related to other people (Cunningham et al., 2008). This again fits with the idea that linking items to the self forms a glue that enhances the integration of information, with the data suggesting that this can arise at multiple levels.

A somewhat different account is as follows. ${ }^{1}$ In the present procedures, every shape was associated with a concept, and thus, for the successful completion of the matching task, the perceptual representation of each shape must be transformed into its associated concept. This shape-to-concept

${ }^{1}$ We thank an anonymous referee for this proposal. interpretation is the same when the stimuli are identical shapes, but it differs (and doubles) when there are two nonidentical shapes. The doubling of the transformation process would lessen the gains from redundant stimuli. The gain from repeating the transformation process for physically identical stimuli may be greatest for self-associated shapes, because self association helps impose the same transformation on the stimuli. Although the effects of self and reward association dissociated in their effects on redundancy gains with identical shapes, both forms of associations affected the redundancy gains with different shapes (and self and reward association did not differ in this respect). The redundancy gains with different shapes might themselves arise at several levels of processing. For example, the gains might reflect both shapes activating a common response (for matching the stimuli), pushing the response over a threshold more quickly than when only a single shape appears. Alternatively, the redundancy gain may stem from the summation of information at a conceptual level, on the basis of the shapes being associated both with the self (see, e.g., Cunningham et al., 2008) and with high reward (Small et al., 2005). This is consistent with previous work showing that, at least under conditions requiring the attentional selection of the target, redundancy gains can be related to the relatively late, attention-related anterior N2 and task-related P3 components in ERP studies, rather than to early ERP components (e.g., the P1) reflecting processing of the physical properties of the stimuli (Akyürek \& Schubö, 2013). These ideas may be tested in the future by more formal analyses that could examine how the redundancy gains come about, but this is beyond the scope of the present experiments, which focused on the dissociation between the self and reward and on the different gains for identical shapes linked to the self.

One point to note here is that we failed to find strong effects of the self and of high reward on the single-shape trials in Experiments 1-3. This might also reflect several factors. One is the contrasts made between the different associations. For example, we used a contrast between the self and the participant's best friend (Exps. 1-2) and a 9:1 reward ratio (Exp. 3). These contrasts may not have been sufficient to differentially affect performance when one item occurred. Sui et al. (2012) found stronger contrasts between self association and a more distant association (self-shape vs. strangershape) than between the self and a close association (friend), consistent with differential familiarity making a contribution to performance. In addition, in Experiments 1-3 we used Chinese rather than Western participants, and the Chinese participants in Experiments 1-2 may have had closer perceived relations to their best friends than do Western individuals (Sui, Liu, \& Han, 2009). This may have lessened the self advantage for single shapes, though the contribution of the self to the redundancy gains led to reliable self-bias effects with both the same and different shapes. In Experiment 4, 
on the other hand, we used Western participants and also increased the contrast between the high- and low-reward stimuli, and here effects of reward bias were found on single shapes (Fig. 4c). To understand which factors are critical will require further research. Nevertheless, in all experiments there were both self and reward advantages with two-item displays, and this was the critical factor for our examination of redundancy gains.

Prior studies have indicated that self-bias effects can be mediated by top-down expectancies. For example, Sui et al. (2014) reported that the self advantage in perceptual matching increases when there is a high probability of self-matching stimuli being present, and they argued that this reflected expectancies for self-related stimuli that were formed across trials. In the present study, we contrasted effects when the critical stimuli were presented simultaneously (e.g., Exps. 2 and 3) and when they were cued (Exps. 1 and 4). With sequential matching, topdown predictions can occur from the label, yet, if anything, in those experiments the different effects of self on the redundancy gains with different shapes were weaker (e.g., Exp. 2). The data indicated that such top-down biases are not necessary for self associations to occur, and in particular they are not necessary for these associations to modulate perceptual grouping.

In conclusion, in the present study we used a redundancy gain paradigm to examine the levels at which the effects of self and reward bias modulate the integration of information. The results indicate that redundancy gains in shape processing are influenced by high-level factors, such as whether stimuli are associated with the self rather than with other people, or with a high rather than a low reward. Self associations modulate redundancy gains even when identical shapes are presented. Reward associations can also modulate redundancy gains, but this was only found when the shapes differed, and not when shapes were identical. This indicates a dissociation between the effects of self and reward bias, suggesting that self-bias effects cannot be reduced simply to the effects of reward. This contradicts a major account of how self-bias effects arise (Northoff \& Hayes, 2011). The data also indicate that reward (along with self bias) modulates higher-level assimilation of information at a conceptual or response level, whereas self bias alone modulates the perceptual integration of shapes.

If self bias does not simply reflect reward, then other factors could be critical — notably, self-related stimuli may carry a higher positive valence than other stimuli (Ma \& Han, 2010), and the valence of the stimulus may be what modulates perceptual processing (e.g., with identical redundant stimuli here). There is evidence that the processing of emotional position stimuli is faster (Kanske \& Kotz, 2011), though there is also evidence for faster processing of stimuli with negative emotional valence (Bradley, Codispoti, Cuthbert, \& Lang, 2001). Whether emotional valence is the critical factor that may lead to the unique perceptual gain for self-related identical stimuli is an important question for future research.
Author note This work was supported by the National Nature Science Foundation of China (Project No. 31371017) and by grants from the Economic and Social Research Council (Grant No. ES/J001597/1, UK) and the European Research Council (Pepe Grant No. 323883).

\section{References}

Akyürek, E. G., \& Schubö, A. (2013). The eletrophysiological locus of the redundant target on visual discrimination in a dual singleton search task. Brain Research, 1537, 180-190. doi:10.1016/j. brainres.2013.09.014

Anderson, B. A., \& Yantis, S. (2013). Persistence of value-driven attentional capture. Journal of Experimental Psychology: Human Perception and Performance, 39, 6-9. doi:10.1037/a0030860

Behrens, T. E. J., Hunt, L. T., Woolrich, M. W., \& Rushworth, M. F. S. (2008). Associative learning of social value. Nature, 456, 245-249.

Bradley, M. M., Codispoti, M., Cuthbert, B. N., \& Lang, P. J. (2001). Emotion and motivation I: Defensive and appetitive reactions in picture processing. Emotion, 1, 276-298. doi:10.1037/1528-3542. 1.3.276

Brédart, S., Delchambre, M., \& Laureys, S. (2006). One's own face is hard to ignore. Quarterly Journal of Experimental Psychology, 59, $46-52$.

Chelazzi, L., Eštočinová, J., Calletti, R., Lo Gerfo, E., Sani, I., Della Libera, C., \& Santandrea, E. (2014). Altering spatial priority maps via reward-biased learning. Journal of Neuroscience, 34, 85948604. doi:10.1523/JNEUROSCI.0277-14.2014

Chelazzi, L., Perlato, A., Santandrea, E., \& Della Libera, C. (2013). Rewards teach visual selective attention. Vision Research, 85, 5872.

Cunningham, S. J., Turk, D. J., Macdonald, L. M., \& Macrae, C. N. (2008). Yours or mine? Ownership and memory. Consciousness and Cognition, 17, 312-318. doi:10.1016/j.concog.2007.04.003

De Greck, M., Enzi, B., Prosch, U., Gantman, A., Tempelmann, C., \& Northoff, G. (2010). Decreased neuronal activity in reward circuitry of pathological gamblers during processing of personal relevant stimuli. Human Brain Mapping, 31, 1802-1812.

Fischer, R., \& Miller, J. (2008). Differential redundancy gain in onset detection versus offset detection. Perception \& Psychophysics, 70, 431-436. doi:10.3758/PP.70.3.431

Hickey, C., Chelazzi, L., \& Theeuwes, J. (2010). Reward changes salience in human vision via the anterior cingulate. Journal of Neuroscience, 30, 11096-11103. doi:10.1523/JNEUROSCI.102610.2010

Holm, S. (1979). A simple sequentially rejective multiple test procedure. Scandinavian Journal of Statistics, 6, 65-70. Retrieved from www. jstor.org/stable/4615733

Humphreys, G. W., Riddoch, M. J., Nys, G., \& Heinke, D. (2002). Unconscious transient binding by time: Neuropsychological evidence from anti-extinction. Cognitive Neuropsychology, 19, 361380 .

Izuma, K., Saito, D. N., \& Sadato, N. (2008). Processing of social and monetary rewards in the human striatum. Neuron, 58, 284-294.

Kanske, P., \& Kotz, S. A. (2011). Positive emotion speeds up conflict processing: ERP responses in an auditory Simon task. Biological Psychology, 87, 122-127.

Keenan, J. P., McCutcheon, B., Sanders, G., Freund, S., Gallup, G. G., \& Pascual-Leone, A. (1999). Left hand advantage in a self-face recognition task. Neuropsychologia, 37, 1421-1425.

Keyes, H., \& Brady, N. (2010). Self-face recognition is characterized by "bilateral gain" and by faster, more accurate performance which persists when faces are inverted. Quarterly Journal of 
Experimental Psychology, 63, 840-847. doi:10.1080/ 17470211003611264

Keyes, H., Brady, N., Reilly, R. B., \& Foxe, J. J. (2010). My face or yours? Event-related potential correlates of self-face processing. Brain and Cognition, 72, 244-254. doi:10.1016/j.bandc.2009.09. 006

Kristjánsson, Á., Sigurjónsdóttir, Ó., \& Driver, J. (2010). Fortune and reversals of fortune in visual search: Reward contingencies for pop-out targets affect search efficiency and target repetition effects. Attention, Perception, \& Psychophysics, 72, 1229-1236. doi:10. 3858/APP.72.5.1229

Lakens, D. (2013). Calculating and reporting effect sizes to facilitate cumulative science: A practical primer for $t$-tests and ANOVAs. Frontiers in Psychology, 4, 863. doi:10.3389/fpsyg.2013.00863

Ma, Y., \& Han, S. (2010). Why respond faster to the self than others? An implicit positive association theory of self advantage during implicit face recognition. Journal of Experimental Psychology: Human Perception and Performance, 36, 619-633.

Miller, J. (1982). Divided attention: Evidence for coactivation with redundant signals. Cognitive Psychology, 14, 247-279. doi:10.1016/ 0010-0285(82)90010-X

Moray, N. (1959). Attention in dichotic listening: Affective cues and the influence instructions. Quarterly Journal of Experimental Psychology, 11, 56-60.

Mordkoff, J. T., \& Danek, R. H. (2011). Dividing attention between color and shape revisited: Redundant targets coactivate only when parts of the same perceptual object. Attention, Perception, \& Psychophysics, 73, 103-112. doi:10.3758/s13414-010-0025-2

Mordkoff, J. T., \& Miller, J. (1993). Redundancy gains and coactivation with two different targets: the problem of target preferences and the effects of display frequency. Perception \& Psychophysics, 53, 527535.

Mordkoff, J. T., Miller, J., \& Roch, A.-C. (1996). Absence of coactivation within the motor component: Evidence from psychophysiological measures of target detection. Journal of Experimental Psychology: Human Perception and Performance, 22, 25-41. doi:10.1037/00961523.22.1.25

Mordkoff, J. T., \& Yantis, S. (1991). An interactive race model of divided attention. Journal of Experimental Psychology: Human Perception and Performance, 17, 520-538. doi:10.1037/0096-1523.17.2.520

Northoff, G., \& Bermpohl, F. (2004). Cortical midline structures and the self. Trends in Cognitive Sciences, 8, 102-107.

Northoff, G., \& Hayes, D. J. (2011). Is our self nothing but reward? Biological Psychiatry, 69, 1019-1025. doi:10.1016/j.biopsych. 2010.12.014

Raab, D. H. (1962). Statistical facilitation of simple reaction times. Transaction of the New York Academy Sciences, 24, 574-590.
Rogers, T. B., Kuiper, N. A., \& Kirker, W. S. (1977). Self-reference and the encoding of personal information. Journal of Personality and Social Psychology, 35, 677-688. doi:10.1037/0022-3514.35.9.677

Rushworth, M. F. S., Noonan, M. P., Boorman, E. D., Walton, M. E., \& Behrens, T. E. (2011). Frontal Cortex and Reward-Guided Learning and Decision-Making. Neuron, 70, 1054-1069.

Serences, J. T. (2008). Value-based modulations in human visual cortex. Neuron, 60, 1169-1181. doi:10.1016/j.neuron.2008.10.051

Shuler, M. G., \& Bear, M. F. (2006). Reward timing in the primary visual cortex. Science, 311, 1606-1609.

Small, D. M., Gitelman, D., Simmons, K., Bloise, S. M., Parrish, T., \& Mesulam, M. M. (2005). Monetary incentives enhance processing in brain regions mediating top-down control of attention. Cerebral Cortex, 15, 1855-1865.

Sui, J., He, X., \& Humphreys, G. W. (2012). Perceptual effects of social salience: Evidence from self-prioritization effects on perceptual matching. Journal of Experimental Psychology: Human Perception and Performance, 38, 1105-1117. doi:10.1037/ a0029792

Sui, J., \& Humphreys, G. W. (2013). The boundaries of self face perception: Response time distributions, perceptual categories, and decision weighting. Visual Cognition, 21, 415-445.

Sui, J., Liu, C. H., \& Han, S. (2009). Cultural difference in neural mechanisms of self-recognition. Social Neuroscience, 4, 402-411.

Sui, J., Rotshtein, P., \& Humphreys, G. W. (2013). Coupling social attention to the self forms a network for personal significance. Proceedings of the National Academy of Sciences, 110, 7607-7612.

Sui, J., Sun, Y., Peng, K., \& Humphreys, G. W. (2014). The automatic and the expected self: Separating self- and familiarity biases effects by manipulating stimulus probability. Attention, Perception, \& Psychophysics, 76, 1176-1184. doi:10.3758/s13414-014-0631-5

Symons, C. S., \& Johnson, B. T. (1997). The self-reference effect in memory: A meta-analysis. Psychological Bulletin, 121, 371-394. doi:10.1037/0033-2909.121.3.371

Tong, F., \& Nakayama, K. (1999). Robust representations for faces: Evidence from visual search. Journal of Experimental Psychology: Human Perception and Performance, 25, 1016-1035. doi:10.1037/ 0096-1523.25.4.1016

Townsend, J. T., \& Nozawa, G. (1995). Spatio-temporal properties of elementary perception: An investigation of parallel, serial and coactive theories. Journal of Mathematical Psychology, 39, 321-359. doi:10.1006/jmps.1995.1033

Yankouskaya, A., Booth, D. A., \& Humphreys, G. (2012). Interactions between facial emotion and identity in face processing: Evidence based on redundancy gains. Attention, Perception, \& Psychophysics, 74, 1692-1771. doi:10.3758/s13414-012-0345-5 